\title{
Dielectric Properties of Fresh Human Blood
}

\author{
Saqib Salahuddin* Lourdes Farrugia $^{\dagger} \quad$ Charles V. Sammut $^{\dagger}$ \\ Martin O'Halloran* Emily Porter*
}

\begin{abstract}
The dielectric properties of blood are extremely important for electromagnetic medical applications such as imaging, hyperthermia and ablation. The aim of this paper is to investigate the dielectric properties of human blood and examine the effects of anti-coagulant agents over a broad frequency range. The measurements were performed using freshly extracted human blood samples with and without the added agents. The measurements were performed within 20 mins after extracting the blood sample. The obtained results demonstrate a significant variation between the dielectric properties of blood with and without anti-coagulant agents. The difference is larger in conductivity than in relative permittivity. The results also demonstrate clear variation in the dielectric properties of female and male blood samples. The findings in this paper suggest that measurements conducted on blood samples with the added agents may not represent the natural in-vivo and inter-patient variations in blood properties. In order to have an accurate representation of in-vivo blood dielectric properties, all factors should be considered carefully.
\end{abstract}

\section{INTRODUCTION}

Blood is a highly functional complex body fluid that serves as the principal transport medium of the body. It delivers oxygen, nutrients, vitamins and metabolites to vital parts of the body and as such is a fundamental part of the immune system. The dielectric properties of blood are extremely important for various medical applications, such as microwave imaging, hyperthermia and ablation [1-6]. These properties reveal various dynamic processes. Moreover, reliable measurement and determination of safe limits for electromagnetic energy absorption by human body is not possible without precise knowledge of dielectric properties of blood $[7,8]$.

A number of research studies have been performed on the dielectric properties of blood [9-13]. Most research has addressed only limited aspects including electrical impedance, erythrocyte suspension, polarization effect, and haematocrit dependency $[14-22]$. Furthermore, most of the studies have been performed using blood containing anticoagulant agents from blood banks. So far, the

\footnotetext{
*Electrical and Electronic Engineering, National University of Ireland Galway, Ireland, e-mail: \{S.salah-ud-din1, Martin.ohalloran, e.porter3\}@nuigalway.ie, tel.: +353 91524411.

$\dagger$ Department of Physics, University of Malta, Malta, e-mail: \{lourdes.farrugia, charles.v.sammut\} @um.edu.mt, tel.: +356 2340 2340, fax: +35623402342.
}

most cited broadband spectra of blood covering several dispersion regions are by Gabriel et al. [23], taken at $37^{\circ} \mathrm{C}$, which is commonly used for specific absorption rate (SAR) calculations and medical purposes. However, the available data is limited in scope and there are are still many unclear aspects of the dielectric properties of blood. This paper aims to investigate the effects of anti-coagulant agents on the relative permittivity and conductivity of human blood over a broad frequency range.

\section{Methodology}

\subsection{Experimental System}

The measurements were performed using the commercially available slim form probe from Keysight Technologies over the frequency range of $400 \mathrm{MHz}-$ $20 \mathrm{GHz}$ with 100 frequency points. The $85070 \mathrm{E}$ dielectric probe kit was connected to the Rohde \& Schwarz ZVA50 vector network analyser (VNA). Three blood sample tubes (all standard vacuum tubes) were selected for the measurements: one without any added agents (i.e., containing pure blood) and two with different anti-coagulant agents (EDTA and sodium citrate). All measurements were conducted at a constant temperature of $37^{\circ} \mathrm{C}$ without moving the probe or the cable. A water bath, a specialized beaker, and a water pump were used to maintain a constant temperature. A customized stand was used in the set-up to support the samples inside the beaker.

\subsection{Acquisition of blood samples}

Blood samples were collected from four different volunteers: one female and three males, in line with local ethical guidelines. For each sample, the three tubes were filled and were immediately placed in the beaker to maintain them at body temperature.

\subsection{Dielectric measurements}

Three measurements were performed at each instance using each blood sample tube and averaged to constitute one measurement. The measurement system was calibrated with a short circuit, open circuit and standard material (de-ionized water). The calibration was validated using $0.9 \% \mathrm{NaCl}$ prior to conducting measurements for each volunteer's 


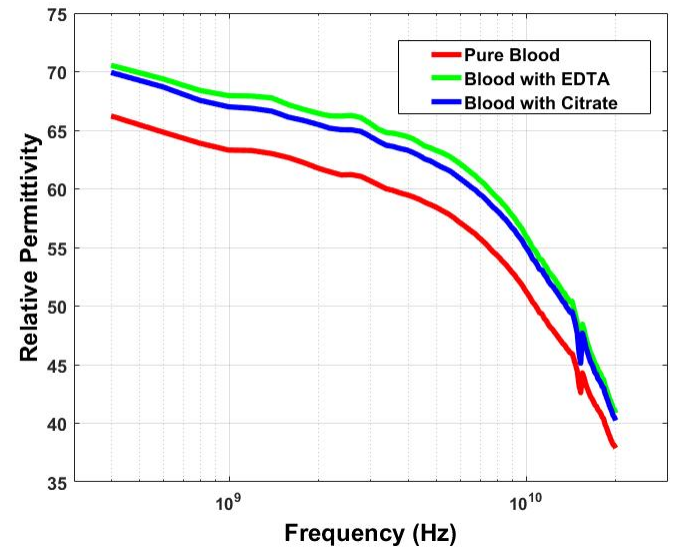

(a) Relative permittivity

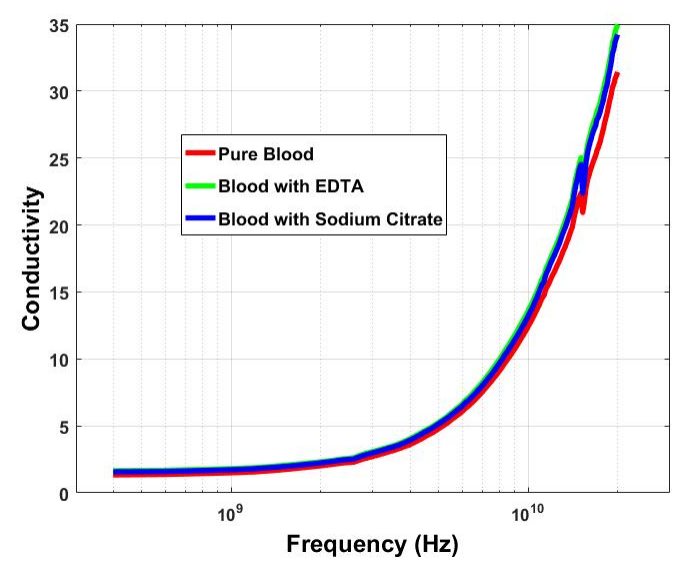

(b) Conductivity

Figure 1: The dielectric properties of female blood sample with and without anti-coagulant agents.

blood sample. The probe was cleaned using alcohol wipes after each measurement. The measurements using each volunteer blood sample were performed within 20 minutes of extraction from the body.

\section{Results}

The relative permittivity and conductivity of the female blood sample with and without the anticoagulant agent are shown in Fig. 1. The figure shows that the relative permittivity values of blood with the two anti-coagulant agents are close to each other but clearly vary from relative permittivity of pure blood with an overall average percentage difference of $8.67 \%$. The average percentage difference in conductivity is $11.29 \%$. The variation is not clearly visible in Fig. 1 (b) because the conductivity values are quite small at low frequencies.

The relative permittivity and conductivity mea-

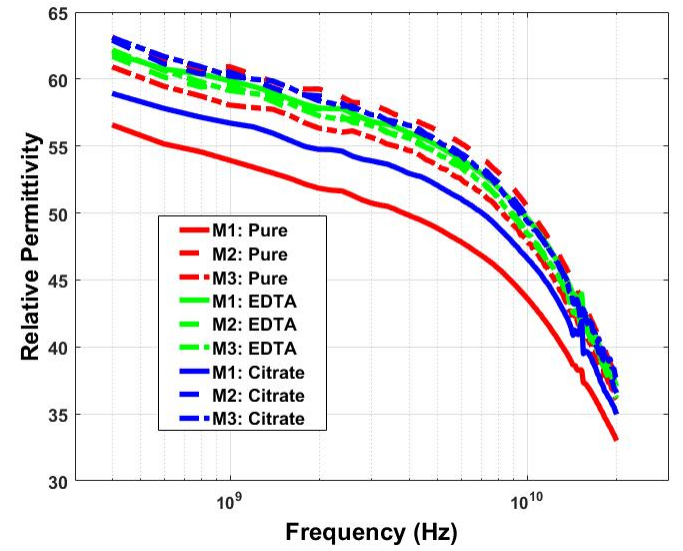

(a) Relative permittivity

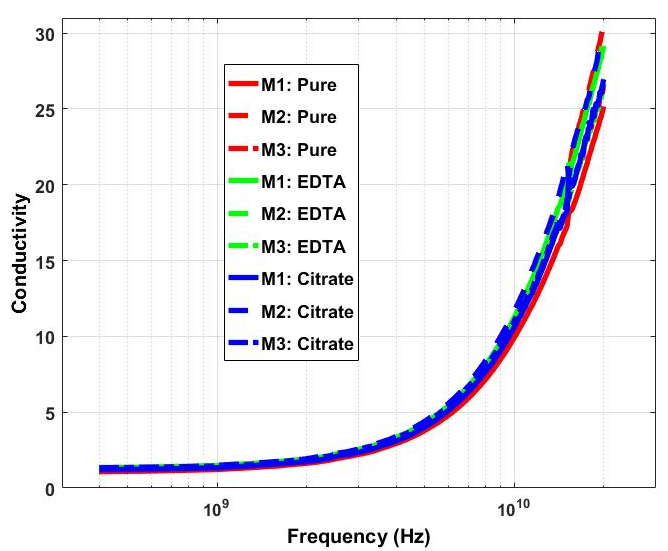

(b) Conductivity

Figure 2: The dielectric properties of male blood samples with and without anti-coagulant agents.

surements of all three male blood samples are illustrated in Fig. 2. The relative permittivity of three male blood samples without the anticoagulant agent has an overall average percentage difference of $14.87 \%$ between the samples. The variation indicates that relative permittivity of human blood is highly dependent on patient. The average difference for conductivity is larger, with a value of $18.81 \%$. The figure shows that the dielectric properties of blood samples with EDTA are close to each other but are not an accurate representative of pure blood properties. The figure also shows that the dielectric properties for sodium citrate vary for different blood samples.

Fig. 3 shows the dielectric properties of a female and a male blood sample without any anticoagulant agent. The figure shows an inter-gender average percentage difference of $17.52 \%$ in relative permittivity and $25.09 \%$ in the conductivity of pure blood. The results are summarised in Table 1. 


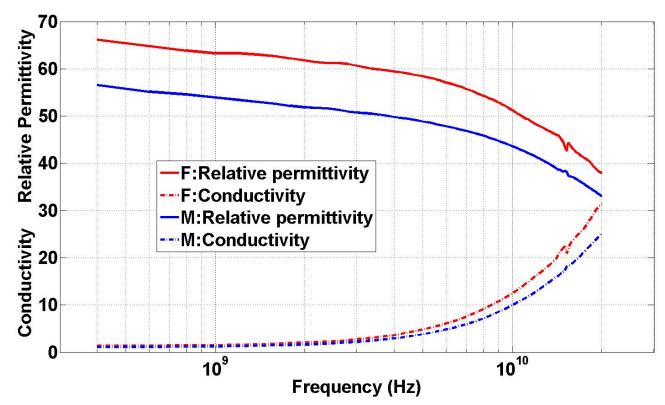

Figure 3: The dielectric properties of female and male blood samples without anti-coagulant agents.

Table 1: Average percentage difference between all blood samples with and without anti-coagulant agents.

\begin{tabular}{l|lccc}
\hline \hline Sample & Pure vs EDTA & Pure vs Citrate & EDTA vs Citrate \\
\hline \multirow{2}{*}{ Female } & Relative Permittivity & 8.67 & 6.82 & 1.70 \\
& Conductivity & 11.29 & 8.32 & 2.66 \\
Male 1 & Relative Permittivity & 12.95 & 6.49 & 5.72 \\
& Conductivity & 15.36 & 7.43 & 6.86 \\
Male 2 & Relative Permittivity & 3.33 & 1.65 & 1.75 \\
& Conductivity & 1.83 & 0.50 & 1.66 \\
Male 3 & Relative Permittivity & 1.69 & 3.55 & 1.83 \\
& Conductivity & 1.79 & 2.67 & 0.98 \\
\hline \hline
\end{tabular}

\section{Conclusion}

In this paper, dielectric measurements were performed and investigated using freshly extracted human blood samples from four different volunteers, one female and three males, over the frequency range of $400 \mathrm{MHz}$ to $20 \mathrm{GHz}$ in order to examine the effects of anti-coagulant agents. The blood sample from each volunteer was placed into three blood sample tubes (all standard plastic vacuum tubes): one without any added agent, i.e. containing pure blood, and two with different anticoagulant agents (EDTA and sodium citrate). The obtained results demonstrate a significant variation between the dielectric properties of blood with and without anti-coagulant agents. The difference is larger in conductivity than in relative permittivity. Further, there is a clear variation in the dielectric properties of female and male blood samples. The results also demonstrate that the dielectric properties differ between different blood samples with same anti-coagulant agent. The investigation in this paper suggests that there are various factors, including the gender and added agents, that affect the dielectric properties of blood and all of these factors should be considered carefully in order to have an accurate representation of in-vivo blood dielectric properties. This research study promises to be a stepping stone towards achieving more accurate dielectric properties of pure human blood, thereby supporting the design and development of microwave imaging and therapeutic devices.

\section{Acknowledgements}

The research leading to these results has received funding from the European Research Council under the ERC Grant Agreement n. 637780: 'BioElecPro'. This work is also supported by the Irish Research Council (grant numbers RCS1325 and RCS1377) and has been developed in the framework of COST Action MiMed (TD1301).

\section{References}

[1] A. Andreano and C. L. Brace, "A comparison of direct heating during radiofrequency and microwave ablation in ex vivo liver," Cardiovascular and interventional radiology, vol. 36, no. 2, pp. 505-511, 2013.

[2] L. Farina, N. Weiss, Y. Nissenbaum, M. Cavagnaro, V. Lopresto, R. Pinto, N. Tosoratti, C. Amabile, S. Assarino, and S. N. Goldberg, "Characterisation of tissue shrinkage during microwave thermal ablation," International Journal of Hyperthermia, vol. 30, no. 7, pp. 419-428, 2014.

[3] X. Nie, Q. Nan, X. Guo, and Z. Tian, "Numerical study of the effect of blood vessel on the microwave ablation shape," Bio-Medical Materials and Engineering, vol. 26, no. s1, pp. S265-S270, 2015.

[4] P. T. Nguyen, A. Abbosh, and S. Crozier, "Microwave Hyperthermia for Breast Cancer Treatment Using Electromagnetic and Thermal Focusing Tested on Realistic Breast Models and Antenna Arrays," IEEE Transactions on Antennas and Propagation, vol. 63, no. 10, pp. 4426-4434, 2015.

[5] T. Sherertz and C. J. Diederich, "Hyperthermia in Locally Recurrent Breast Cancer," in Radiation Therapy Techniques and Treatment Planning for Breast Cancer, 2016, pp. 145158.

[6] P. T. Nguyen, "Focusing microwave hyperthermia in realistic environment for breast cancer treatment," p. 156, 2016.

[7] IEEE Standards Coordinating Committee, IEEE Standard for Safety Levels with Respect to Human Exposure to Radio Frequency Electromagnetic Fields, 3kHz to 300 GHz, 1992. 
[8] B. B. Beard, W. Kainz, T. Onishi, T. Iyama, S. Watanabe, O. Fujiwara, J. Wang, G. BitBabik, A. Faraone, and J. Wiart, "Comparisons of computed mobile phone induced SAR in the SAM phantom to that in anatomically correct models of the human head," IEEE Transactions on Electromagnetic Compatibility, vol. 48, no. 2, pp. 397-407, 2006.

[9] J. Krupa, K. B, and J. Terlecki, "Method of calculating conductivity of human erythrocytes interior based on measurement of electric magnitudes of suspension," Biophysik, vol. 8, no. 3, p. 227, 1972.

[10] M. R. Willis, Dielectric and electronic properties of biological materials, 1980, vol. 8, no. 1.

[11] R. Pethig, "Dielectric properties of biological materials: biophysical and medical applications," IEEE Transactions on Electrical Insulation, no. 5, pp. 453-474, 1984.

[12] J. M. Alison and R. J. Sheppard, "Dielectric properties of human blood at microwave frequencies." Physics in medicine and biology, vol. 38, no. 7, pp. 971-978, 1993.

[13] Y. Feldman, I. Ermolina, and Y. Hayashi, "Time domain dielectric spectroscopy study of biological systems," IEEE transactions on dielectrics and electrical insulation, vol. 10, no. 5, pp. 728-753, 2003.

[14] H. Schwan, "Electrical properties of blood and its constitutents: Alternating current spectroscopy," Blut, vol. 46, no. 4, pp. 185-197, 1983.

[15] J.-Z. Bao, C. C. Davis, and M. L. Swicord, "Microwave dielectric measurements of erythrocyte suspensions," Biophysical journal, vol. 66, no. 3, p. 2173, 1994.

[16] R. Lisin, B. Z. Ginzburg, M. Schlesinger, and Y. Feldman, "Time domain dielectric spectroscopy study of human cells. I. Erythrocytes and ghosts," Biochimica et Biophysica Acta (BBA)-Biomembranes, vol. 1280, no. 1, pp. 34-40, 1996.

[17] T. Chelidze, "Dielectric spectroscopy of blood," Journal of Non-Crystalline Solids, vol. 305, no. 1-3, pp. 285-294, 2002.

[18] F. Jaspard, M. Nadi, and a. Rouane, "Dielectric properties of blood: an investigation of haematocrit dependence." Physiological measurement, vol. 24, no. 1, pp. 137-147, 2003.
[19] Y. Hayashi, I. Oshige, Y. Katsumoto, S. Omori, A. Yasuda, and K. Asami, "Dielectric inspection of erythrocyte morphology," Physics in medicine and biology, vol. 53, no. 10, p. 2553, 2008.

[20] Y. Zhang, L. Zhong, S. Tan, C. Xu, A. Preparation, and C. Suspension, "Dielectric Properties of Red Blood Cell Suspensions Based on Broadband Dielectric Spectrum," Physics, no. 50277030, pp. 3-6, 2010.

[21] S. Abdalla, S. S. Al-ameer, and S. H. AlMagaishi, "Electrical properties with relaxation through human blood," Biomicrofluidics, vol. 4, no. 3, pp. 1-16, 2010.

[22] A. Rauf, "a Dielectric Study on Human Blood and Plasma," International Journal of Science, Environment and Technology, vol. 2, no. 6, pp. 1396-1400, 2013.

[23] S. Gabriel, C. Gabriel, and R. W. Lau, "The dielectric properties of biological tissues: III. Parametric models for the dielectric spectrum of tissues." Physics in medicine and biology, vol. 41, no. 11, pp. 2271-2293, 1996. 